\title{
Ein pankratisches Fernrohr
}

\author{
von \\ F. C. Donders.
}

Ein holländisches oder Galilaeisches Fernrohr besteht bekanntlich aus einem negativen Ocular, mit der Brennweite $f$, und einem positiven Objectiv, mit der Brennweite $f+1$. Dieses 1 bedeutet die Intfernung zwischen Ocular and Objectiv, bei welcher die Brennweite des Fernrohrs unendlich wird, das heisst, wodurch parallel auf das Objectiv fallende Strahlen parallel aus dem Ocular in das Auge treten.

Die Vergrösserung ist $m=\frac{f+1}{f .}$

Ein derartiges Fernrohr hat auf die Weise, bei der Brennweite $\mathrm{F}=\infty$, eine constante Länge und eine constante Vergrösserung.

Mein Streben war nun dahin gerichtet, ein Fernrohr zu construiren, wodurch alle Vergrösserungen in einer continuirlichen Reihe durch Verschiebung der Gläser innerhalb bestimmter Grenzen zu erzielen seien. Für ein derartiges Fernrohr scheint mir die Bezeichnung ,pankratisches" nicht unzutreffend.

Die nächste Veranlassung zu dieser Construction lag in dem Bedürfniss versehiedene Vergrösserungen zu erhalten 
zur Vergleichung der Grössenwerthe, unter welchen dasselbe Object sich bei Augen verschiedener Refraction darstellt. Refractionsverschiedenheit (Anisometropie) kommt in den beiden Augen desselben Individuums nicht selten vor. Liegt der Grund dieser Verschiedenheit nicht in der Verschiedenheit des dioptrischen Systems, sondern, wie in der Regel, fast ausschliesslich in den verschiedenen Längen der Sehachsen, dann ist, nach Correction der Ametropie durch ein im vorderen Brennpunkt angebrachtes Glas, das Netzhautbild, wio Knapp bewiesen hat, von gleicher Grösse wie das des emmetropischen Auges. In diesem Fall müssen daher die Netzhautbilder von demselben Object, falls die Ametropie corrigirt ist, auf beiden Augen gleich gross sein. Es entsteht nun die Frage, in welcher relativen Grösse wir alsdann mit jedem Auge das Object sehen. Durch ein Prisma vor einem Auge, besser noch durch ein sehr schwaches Prisma vor jedem Auge, das eine mit der brechenden Kante nach oben, das andere nach unten, erscheint das eine Halbbild über dem anderen und können die Grössen derselben untereinander verglichen werden. Als Object wähle man eine flache Figur, ein Quadrat, Dreieck oder einen Kreis.

In dieser Weise zeigte sich sofort, dass in der Regel das Object vor einem myopischen Auge kleiner, vor einem hypermetropischen grösser erscheint als vor einem emmetropischen. Mir lag aber daran, das richtige Verhältniss dieser Grössen kennen zu lernen und dieser Zweck sehien mir allein erreichbar durch die genaue Bestimmung, durch welche Vergrösserung des kleineren oder Verkleinerung des grösseren Bildes beide völlig gleich würden. Die gewünschte Vergrösserung und Verkleinerung kann nun erreicht werden durch ein schwaches Fernrohr, welches vor ein Auge gehalten wird. Dazu bediente ich mich zweier Ringe in einem Abstand von 2 Pariser Zoll, in welche die Gläser des Brillenkastens in der Differenz von 2 Zoll Brennweite 
hineingesetzt wurden, also Combinationen von $-\frac{1}{2}$ mit $+\frac{1}{4},-\frac{1}{3}$ mit $+\frac{1}{5}$ u. s. w. bis $-\frac{1}{28}$ mit $+\frac{1}{30}$. Bei der ersten war $\mathrm{m}=2$, bei der letzten $\mathrm{m}=\frac{15}{14}$. Hatte man die Combination gefunden, die das kleinste Halbbild dem grössten gleich machte, dann musste durch Umkehrung des Fernrohrs, welches nun vor das andere Auge gehalten wurde, das grösste Halbbild dem kleinsten gleich werden. Darin hatte man eine sichere Controle. Indess waren mit dieser Methode grosse Schwierigkeiten verbunden. Bei vergleichenden Prüfungen verschiedener Combinationen schwankte man in der Wahl; die steten Nachprüfungen waren zeitraubend, das Resultat unsicher und nicht genau. Ueberdies war das Minimum der Vergrösserung, welches durch die verfügbaren Gläser zu erreichen war, in vielen Fällen zu. gross. Es war klar, dass ein Fernrohr, dessen Vergrösserung, unter Beibehaltung seines Focus auf $\infty$, innerhalb gegebener Grenzen variiren kann, besser zum Ziele führen musste, und deshalb stellte ich mir die Frage, ob sich ein derartiges Fernrohr construiren liesse.

Sehr bald überzeugte ich mich, dass rexschiedene Combinationen von drei und mehr Linsen dazu verwendbar waren. Unter diesen wählte ich eine Combination yon 3 Linsen und zwar folgende: I. ein feststehendes concaves Ocular; III. ein bewegliches concaves Objectiv, und zwischen diese; II. eine verschiebbare Convexlinse, die stärker war als I oder II, aber schwächer als I $+\mathrm{II}$.

Eis war mir zunächst darum zu thun, Grenzwerthe von $m=\frac{4}{3}$ bis $m=\frac{3}{4}$ aurch ein kurzes Fernrohr zu erhalten. Dem würde genügt, wenn die Werthe von I, II, III resp. 12, 21 und 12 Dioptrien betrügen, das heisst, wenn die- 
selben eine Brennweite hätten von $\frac{1}{12}, \frac{1}{21}$ and $\frac{1}{12}$ Meter. Wird dann II mit III vereinigt, dann erhält man ein positives Objectiv von $21-12=9$, ein negatives Ooular von 12 und folglich $m=\frac{12}{9}$. Wird II mit I vereinigt, dann erhält man ein positives Ocular von $21-12=9$, ein negatives Objectiv von 12 , folglich $\mathrm{m}=\frac{9}{12}$. Die Länge des Fermrohrs muss bei dieser Vergrösserung $\frac{1}{9}-\frac{1}{12}=$ 0,027 Meter betragen. Indem ich in Betracht zog, dass II nicht mit III vereinigt, sondern nur an einander gerückt werden kann und daher die Grenzwerthe von $m$ nicht $z u$ erhalten sind, so wählte ich $\mathrm{I}=12, \mathrm{II}=20, \mathrm{III}=12 \mathrm{Di}-$ optrien, welche $\mathrm{m}=\frac{12}{8}$ bis $\mathrm{m}=\frac{8}{12}$ repräsentiren, in Wirklichkeit aber ungefähr $\frac{4}{3}$ bis $\frac{3}{4}$ geben.

Bei der Anwendung ging ich aus von $\mathrm{m}=1$ und $\mathrm{F}$ $=\infty$.

Eig. 1 .

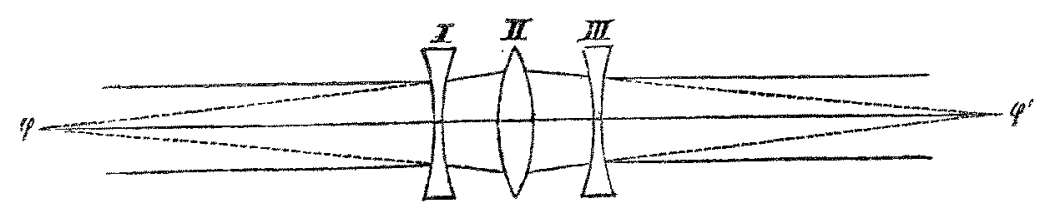

Die Linse II steht dabei in der Mitte zwischen I und III, die so weit auseinander liegen, dass ihre Brennpunkte $\varphi$ und $\varphi^{t}$ zugleich die conjugirten Brennpunkte von II sind. $\mathrm{L}$ ist dann $=33,3 \mathrm{~m}, \mathrm{~m}$. Schiebt man nun II zurück, dann wird $m>1$, aber sofort wird anch das Bild undeutlich: soll nämlich $\mathrm{F}=\infty$ bleiben, dann muss auch III zurückgeschoben werden, anfangs langsam, nach und nach schneller, 
so dass bei $m=\frac{3}{2}$, dem Maximum der Vergrösserung, 1 $=\frac{1}{8}-\frac{1}{12}$ Meter $=125-83,33=41,66$ beträgt.

Für den minder Geübten verursacht die Verschiebung der beiden Linsen practisch einige Schwierigkeiten. Hat man durch Versehiebung von II ungefähr die verlangte Grösse erhalten, dann muss man III verschieben, bis das Bild scharf wird, darauf wieder II, für die verlangte Grösse, und erforderlichen Falls III zum zweiten Mal. Ich stellte mir nun die Frage, ob diese Schwierigkeiten nicht dureh eine bestimmte Combination zu eliminiren wăren, wobei ich sofort erkannte, dass sie wenigstens auf ein Minimum zu reduciren sind, indem III und I verschiedene Werthe erhalten. Für $m=1$ kommt II dann nicht mehr in die Mitte von I und III zu stehen, und beim Zurückschieben von II, wobei $\mathrm{m}>1$ wird, muss III sich anfänglich ein wenig an I nähern, um erst bei weiter zunehmender Vergrösserung sich aufs Neue von I zu entfernen. Wir erhalten daher in diesem Falle mit dem Steigen der Werthe von $\mathrm{m}$ einen Wendepunkt für die Werthe von $\mathrm{l}$. An Stelle $\begin{array}{llll}\text { von } & 12, & 20, & 12, \\ \text { setzte ich nun } & 12, & 20, & 11, \\ \text { und später } & 12, & 19, & 11,\end{array}$ welche Combination sich in der That für kleine Vergrösserungen ganz vortrefflich eignete.

Um für alle erforderlichen Berechnungen bequeme Formeln zu erhalten und wenn möglich Angabe eines noch besseren Systems, wandte ich mich an meinen verehrten Freund und Collegen Grinwis, welcher, bereitwillig wie immer, mir folgendes übersandte:

III

Brennweite Ocular. Mittellinse. Objectiv.

Es sei:

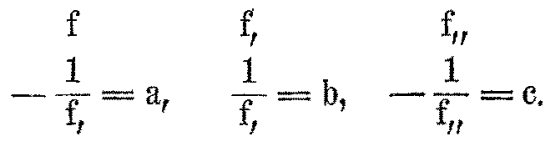


Ferner die Entfermung I zu II (Ocular zur Mittellinse) $=\Delta$ II zallI (Mittellinsezum Objectiv) $=\Delta^{\prime}$ 1) Wenn $\triangle$ oder $\Delta^{\prime}$ bekannt sind, die Vergrösserung $m$ zu finden:

$$
\begin{aligned}
& m=\frac{b-a+a b \Delta}{c} \ldots \ldots . . . \\
& m=\frac{a}{b-c+b c \Delta^{\prime}} \ldots . . .
\end{aligned}
$$

2) Wenn $m$ gegeben ist, $\Delta$ und $\Delta^{\prime}$ zu finden;

$$
\begin{aligned}
& \Delta=\frac{c m-(b-a)}{a} \ldots . . . . \\
& \Delta^{d}=\frac{a-(b-c) m}{b c m} \text {. . . . . . . }
\end{aligned}
$$

3) Wenn $m$ gegeben ist, die Länge 1 ron dem ganzen System zu finden:

$$
I=\frac{c^{2} m^{2}-(b c-2 a c+a b) m+a^{2}}{a b c m} . .
$$

In erster Linie gebranchte ich nun diese Formeln zux Berechnung von $\Delta$ und $\Delta^{\prime}$ und von $1=\Delta+\Delta^{\prime}$, fürr die verschiedenen Werthe von $m$ in dem System $a=12, b=20$, $c=12$ und fand:

$\begin{array}{lclc}\mathrm{m} & \Delta & \Delta^{\prime} & 1 \\ 1,5 & 41,66 & 0 & 41,66 \\ 1,4 & 36,66 & 2,381 & 39,05 \\ 1,3 & 31,66 & 5,125 & 36,79 \\ 1,2 & 26,66 & 8,333 & 35,00 \\ 1,1 & 21,66 & 12,121 & 33,69 \\ 1, & 16,66 & 16,66 & 33,33 \\ 0,9 & 11,66 & 22,222 & 33,89 \\ 0,8 & 6,66 & 29,16 & 35,83 \\ 0,7 & 1,66 & 38,095 & 39,76 \\ 0,66 & 0, & 41,66 & 41,66\end{array}$

Das Resultat dieser Berechnung babe ich in Figur 2 niedergelegt. Die Abscisse I I, anf der die Werthe von $m$ verzeichnet sind, repräsentirt zugleich die constante Lage 
Fig. 2。

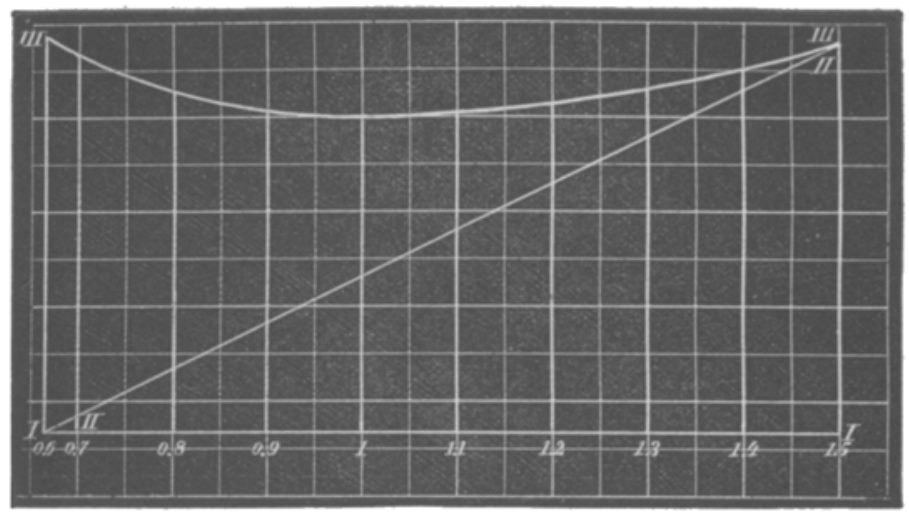

von I. Die Tinien II II und III III geben die Lage von II und III an für alle Werthe von $m$. So liefern uns die Ordinaten der Figur die Lage der Gläser und daher die Werthe von $\Delta, \Delta^{\prime}$ und 1 für alle Werthe von $\mathrm{m}$ in den wirklichen Abmessungen des Fernrohrs.

Man sieht, dass II für gleiche Veränderung von $\mathrm{m}$ überall gleiche Lageveränderung erfährt, d. h., dass die arithmetischen Veränderungen der Werthe von $m$ mit den Verschiebungen in gleichem Verhältniss stehen und dass daher die Vergrösserungen auf einer proportional eingetheilten Scala abgelesen. werden können.

Weiter sieht man, dass für kleine Abweichungen von $\mathrm{m}=1$ nur geringe Verschiebung von III erforderlich ist, aber dass diese bei $\mathrm{m}=1,2$ bereits 1,66 beträgt: wird die Verschiebung nicht gemacht, dann bekommt das Fernrohr eine negative Brennweite von 2,86 Meter, wobei die Bilder nichts weniger als scharf werden. Auf die Bedeutung hiervon kommen wir später zurück.

Für Systeme, wie das hier beschriebene, ist das Maximum der Vergrösserung

$$
\mathrm{M}=\mathrm{a}:(\mathrm{b}-\mathrm{c}),
$$


das Maximum der Länge $\mathrm{L}=\frac{1}{\mathrm{~b}-\mathrm{c}}-\frac{1}{\mathrm{a}}$

$$
\begin{gathered}
\text { Ist } a=12, b=13, c=12 \\
\text { dann ist } M=12: 1=12 \\
L=1000-83,33=916,66 \mathrm{~mm} . \\
\text { Ist } a=12, b=23, c=12 \\
\text { dann ist } M=12: 11=1,0909 \\
L=90,9-83,3=7,6 \mathrm{~mm} .
\end{gathered}
$$

Hierdurch nähern wir uns den Grenzen von $b$ in Bezug auf $a$ und c. Ist $b=0$ dann werden $M$ und $L$ unendlich. Ist $\mathrm{b}=2 \mathrm{c}$, dann ist $\mathrm{M}=1$ und $\mathrm{L}=0$.

Verlangt man furr gleiche Grenzwerthe von $M$ längere oder kürzere Fernrohre, dann lat man die Werthe von a, $b$ and $c$ nur in gleichem Verhaltniss zu verkleinem oder za vergrössern.

In zweiter Linie haben wir die Formeln angewandt auf das System $a=12, b=19, c=11$ und dafür gefunden:

$\begin{array}{lccl}1,5 & 41,6 & 0 & 41,66 \\ 1,4 & 36,84 & 2,734 & 39,574 \\ 1,3 & 32,02 & 5,846 & 37,866 \\ 1,2 & 27,19 & 9,57 & 36,76 \\ 1,19\left(-\frac{12^{2}}{11^{2}}\right) & 26,71 & 9,97 & 36,68 \\ 1,1 & 22,37 & 13,92 & 36,29 \\ 1,0909\left(=\frac{12}{11}\right) & 21,93 & 14,35 & 36,28 \\ 1, & 17,54 & 19,14 & 36,68 \\ 0,9 & 12,72 & 25,52 & 38,24 \\ 0,8 & 7,89 & 33,43 & 41,32 \\ 0,7 & 3,07 & 43,75 & 46,82 \\ 0,6363 & 0 & 51,95 & 51,95\end{array}$


Figur 3 stellt dieses Resultat dar:

Fig. 3.

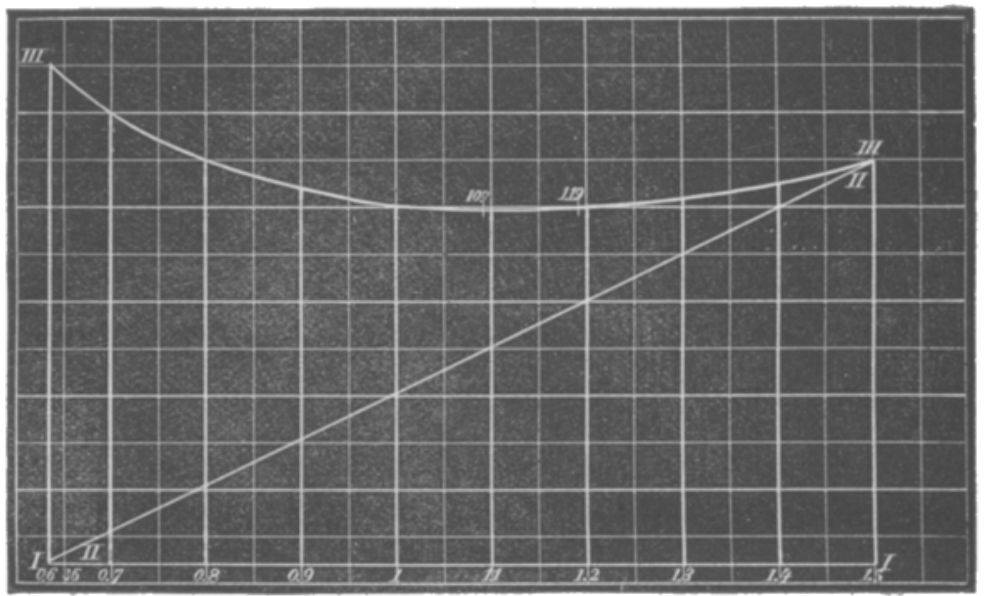

Vergleicht man Fig. 2 und 3, so tritt ein bemerkenswerther Unterschied zu Tage. Zwar steigt $m$ wieder gleichmässig mit $\triangle$ und mit dem Steigen von $m$ nimmt 1 $=\Delta+\Delta^{\prime}$ erst ab und dann wieder $\mathrm{zu}$; allein $\mathrm{m}=1$ făllt, wie ich vorausgesehen, nicht mehr mit dem Minimum von 1 zusammen, das erst bei, $\mathrm{m}=\frac{\mathrm{a}}{\mathrm{c}}$, d. i. bei $\mathrm{m}=\frac{12}{11} \mathrm{er}-$ reicht wird. Zu beiden Seiten von dem Minimum finden wir num jedesmal zwei übereinstimmende Werthe von l, und zwar für $\mathrm{m}=\frac{\mathrm{a}^{2}}{\mathrm{~b}^{2}}=\frac{144}{121}=1,19$, gleiche Werthe als für $m=1$. Dieses weicht noch wenig von dem Minimum ab.

$$
\begin{array}{rlrl}
\mathrm{m} & =1 & 1 & =36,68 \\
\mathrm{~m} & =\frac{12}{11}, \text { Minimum von } \mathrm{l} & =36,28 \\
\mathrm{~m} & =\frac{144}{221} & 1 & =36.68
\end{array}
$$

Der Unterschied beträgt nur $0,4 \mathrm{~mm}$.

Dies bedeutet, dass man von $m=1$ bis $m$ 
$=\frac{144}{121}$, um dabeì $F=\infty$ zu erhalten, die objectivlinse nur um $0,4 \mathrm{~mm}$. za verschieben braucht.

Es fragt sich, ob die Versehiebung wirklich elforderlich ist, oder ob man innerhalb der angegebenen Grenzen von $m$ nicht mit der Verschiebung der Mittellinse allein auskommt.

Um darüber ein Urtheil zu gewinnen, berechnete ich die Brennweite von dem System in dem Falle, dass III nicht verschoben war. Bei der geringsten Länge des Systems, entsprechend einer Vergrösserung von $\frac{12}{11}$, ist $\Delta=21,93, \Delta$ $=14,35,1=36,28$. Geht man nun aus von $\mathrm{m}=1 \mathrm{mit}$ $F=\infty$, wobei $I=36,68$, und verschiebt man lediglich die Mittellinse, so erhält man $\Delta=21,93, \Delta^{\prime}=36,68-21,93$ $=14,75$. Bei dieser Stellung der Gläser fand ich nun durch Berechnung eine positive Brennweite $F^{\prime}=17,3$ Meter. Diese bleibt für die Schärfe der Bilder so gut wie unmerklich and darf ebenfalls in Bezug auf die Vergrösserung wohl unberücksichtigt bleiben. Dasselbe gilt von der negativen Brennweite von 20 Meter, die ich für $\Delta=28,639$ und $\Delta^{\prime}=1,049,1$ constant $=36,68$, berechnete, wobei $\Delta$ einer Vergrösserung von $\mathrm{m}=1,23$ entspricht. Das System kann daher von $m=1$ bis zu $m=1,23$ und, mittelst Umkehrung, von $\mathrm{m}=1$ bis zu $\mathrm{m}=0,813$ gebraucht werden, ohne dass es nöthig ist die Objectivlinse zu verschieben. Diesen wichtigen Vortheil erreicht man dadurch, dass man I und III nicht gleiche Werthe gieht. Zu dem Zweck, zu welchem ich das pankratische Fernrohr zunächst construirte, brauchte ich die angeführten Grenzen fast nie zu überschreiten.

Ausserbalb dieser und, wo es auf grosse Genauigkeit ankommt, auch innerhalb dieser Grenzen wird man gut thun, $F=\infty$ zu nehmen. Es könnte wohl ein Mechanismus angebracht werden, durch welchen zugleich mit der Ver- 
schiebung von II die entsprechende Stellung von III erreicht würde, wobei dann auch $\mathrm{a}=\mathrm{c}$ sein könnte. Allein es genügt auch eine doppelte Scala anzubringen, eine um die Stellung von II, die andere, um die entsprechende Stellung von III abzulesen: je nachdem dann der Beobachter II verschiebt, um die erforderliche Vergrösserung aufzusuchen, kann man III die entsprechende Stellung geben. Fiür die Mittellinse ist die Vertheilung eine gleichmässige (vid. II II, Fig. 3); wie sie für die Objectivlinse sein muss, ergiebt sich aus der Curve III III.

Dass innerhalb der gewöhnlichen Grenzen von $m$ die Verschiebung von III so gering ist, bewirkt, dass das Bild nie aufhört scharf zu sein, wodurch jede Schwierigkeit für das Aufsuchen der erforderlichen Vergrösserung weggenommen ist. Hat man dieselbe gefunden, dann kann der Beobachter selber III, soweit erforderlich, nach II hin verschieben und, indem er II nochmals einstellt, das Resultat jedenfalls für genau erachten. Um alle Vortheile eines solchen Systems zu haben, sei im Allgemeinen a : $c=$ der Quadratwurzel aus der Vergrösserung, welche man nur ausnahmsweise zu überschreiten braucht.

Die veriangte absolute Grenze von $\mathrm{m}$ erhält man durch die Wahl von $b$ (siehe oben). Mit $b$ ändert sich nun auch die Brennweite $\mathrm{F}$, die das System bei $\mathrm{m}=\frac{\mathrm{a}}{\mathrm{c}}$ erhält und zwar so, dass in den Systemen, die für meinen Zweck in Betracht kommen, $F$ ungefähr in gleichem Verhältniss $\mathrm{zu} \mathrm{b}$ steht. Ich fand nämlich für:

$$
\begin{aligned}
& a=12 \quad b=20 \quad c=11 \quad \mathrm{~F}=18,386 \mathrm{M} \text {. } \\
& a=12 \quad b=19 \quad c=11 \quad F=17,307 \quad M \text {. }
\end{aligned}
$$

Die Länge des Fernrohrs bestimmt man nach Belieben, indem man, mit Erhaltung der relativen, die absoluten Werthe von $a, b$ and $c$ verändert, $z a$ welchen die Länge des Fermohrs im umgekehrten Verhältniss steht. Mit der Verlängerung des Fernrohrs ist der Vortheil verknüpft, dass 
F grösser wird und die Bilder, wo die Verschiebung von III unterbleibt, desshalb weniger an Schärfe verlieren.

Das pankratische Fermrohr lässt sich noch zu anderen Zwecken gebrauchen und das Princip kann auf andere dioptrische Systeme übertragen werden. Ich finde vielleicht Veranlassung später darauf zurückzukommen.

Nachschrift. Meine Beschreibung des pankratischen Fermohrs in der "Kon. Alrademie van Wetenschappen" hat zu einer Mittheilung von Prof. Bosscha (Proces-verbaal vom 29. September) Veranlassung gegeben. Dieselbe lautet folgendermassen:

„Herr Bosscha zeigt das Modell eines kleinen Apparates, der dazu bestimmt ist, das sogenannte pankratische Fermrohr von Herrn Donders der Bedingung genügen zu lassen, dass das optische System immerhin wirklich ein Fernrohr sei. Dazu muss der Fokalabstand unendlich gross sein. Wenn $\varphi_{1} \varphi_{2} \varphi_{3}$ die Fokalabstände von drei Linsen sind, $\Delta_{1}$ der Abstand der ersten zwei Linsen, $\Delta_{2}$ der der zweiten und dritten, dann ist der Fokalabstand $f$ des Systems bestimmt durch das Verhältniss

$$
\begin{gathered}
-\frac{1}{f}=-\frac{1}{\varphi_{1}}-\frac{1}{\varphi_{3}}-\frac{1}{\varphi_{3}}+\Delta_{1}\left(\frac{1}{\varphi_{1} \varphi_{2}}+\frac{1}{\varphi_{1} \varphi_{3}}\right)+\Delta_{2} \\
\left(\frac{1}{\varphi_{1} \varphi_{3}}+\frac{1}{\varphi_{2} \varphi_{3}}\right)-\frac{d_{1} d_{2}}{\varphi_{1} \varphi_{2} \varphi_{3}}
\end{gathered}
$$

Dies kann, wenn $\frac{1}{\mathrm{f}}=0$ ist, auch so geschrieben werden:

$$
\left[\Delta_{1}-\left(\varphi_{1}+\varphi_{2}\right)\right]\left[\Delta_{2}-\left(\varphi_{2}+\varphi_{3}\right)\right]=\varphi_{2}^{2}
$$

Dieser Bedingung kann durch die sogenannte Raute von Peaucellier genügt werden. Setzt man die zweite Linse auf das Gelenk welches die beiden Querarme verbindet, die erste Linse in einer Entfernung $-\varphi_{1}-\varphi_{2}$ einwärts von einem der Eckpunkte, die dritte in einem $\mathrm{Ab}$ stand $-\varphi_{2}-\varphi_{3}$ einwärts rom gegenüberliegenden Eckpunkt, (wobei vorausgesetzt ist, dass die eben genannten 
Querarme die beiden anderen Eekpunkte verbinden), dann werden die drei Linsen sich so bewegen, dass das System, wenn es in einer der Stellungen ein Fernrohr ist, in allen anderen diese Eigenschaft behält. Dieselbe Raute kann für andere optische Systeme dienen, wenn nux die mittlere Linse dieselbe bleibt. Man hat dann nur die Abstände $-\varphi_{1}-\varphi_{2}$ und $-\varphi_{2}-\varphi_{3}$ gehörig za ändern.

In derselben Sitzung reichte Herr Ou demans, Professor der Astronomie in Dtrecht, eine Abhandlung ein "sur la théorie de la lunette pancratique de Mr. Donders", die in den "Verslagen en mededeelingen etc." des laufenden Jahres erscheinen wird. 